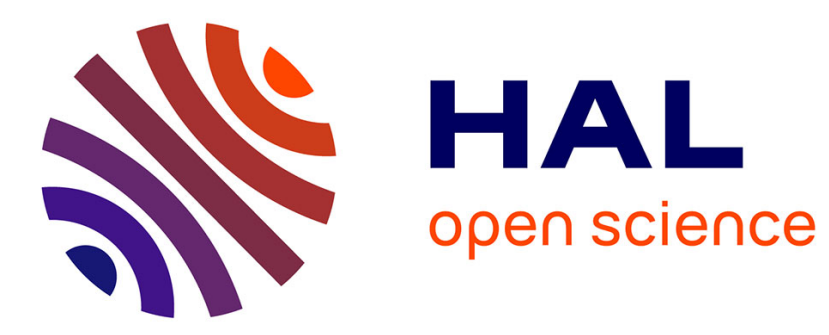

\title{
Odor discrimination in terrestrial and aquatic environments in California sea lions (Zalophus californianus) living in captivity
}

Jules Brochon, G. Coureaud, Cyril Hue, Bérénice Crochu, Isabelle Charrier

\section{- To cite this version:}

Jules Brochon, G. Coureaud, Cyril Hue, Bérénice Crochu, Isabelle Charrier. Odor discrimination in terrestrial and aquatic environments in California sea lions (Zalophus californianus) living in captivity. Physiology \& behavior, 2021, 235, pp.113408. 10.1016/j.physbeh.2021.113408 . hal-03367320v1

\section{HAL Id: hal-03367320 \\ https://cnrs.hal.science/hal-03367320v1}

Submitted on 6 Oct 2021 (v1), last revised 8 Oct 2021 (v2)

HAL is a multi-disciplinary open access archive for the deposit and dissemination of scientific research documents, whether they are published or not. The documents may come from teaching and research institutions in France or abroad, or from public or private research centers.
L'archive ouverte pluridisciplinaire HAL, est destinée au dépôt et à la diffusion de documents scientifiques de niveau recherche, publiés ou non, émanant des établissements d'enseignement et de recherche français ou étrangers, des laboratoires publics ou privés. 
1 Odor discrimination in terrestrial and aquatic environments in California sea

2 lions (Zalophus californianus) living in captivity

3

4 Jules Brochon ${ }^{1}$, Gérard Coureaud ${ }^{2}$, Cyril Hue $^{3}$, Bérénice Crochu ${ }^{3}$, Isabelle Charrier ${ }^{1}$

5

61 Université Paris-Saclay, Université Paris-Sud, CNRS, UMR 9197, Institut des $7 \quad$ Neurosciences Paris-Saclay, 91405 Orsay, France.

$8 \quad{ }^{2}$ Centre de Recherche en Neurosciences de Lyon, Bron, France

$9 \quad{ }^{3}$ Zoo de La Flèche, La Flèche, France

Corresponding Authors at: Institut des Neurosciences Paris Saclay (NeuroPSI), UMR 9197 CNRS, Université Paris Sud, 91405 Orsay, France (Isabelle Charrier) and at Centre de Recherche en Neurosciences de Lyon (CRNL), INSERM U1028, CNRS UMR 5292,

15 Université Claude Bernard Lyon 1, Université Jean Monnet, CH Le Vinatier Bâtiment Neurocampus, 95 Boulevard Pinel, 69675 Bron cedex (Gérard Coureaud). E-mail addresses: isabelle.charrier@u-psud.fr, gerard.coureaud@cnrs.fr 
20

Pinnipeds, as any mammal species, use multimodal signals, including olfactory ones, to ensure vital functions. Thus, some pinniped species seem able to use olfaction in both social and foraging contexts and to discriminate between different odors in air including both natural and artificial odors, but studies on that topic remain scarce. Here, we studied the olfactory capabilities of California sea lions living in captivity at La Flèche Zoo (France) in both terrestrial and aquatic environments. We used two categories of odors: social odors (from familiar individuals of the same group, unfamiliar individuals from another Zoo, animal zookeepers and a terrestrial carnivore) and non-social odors (food and odors identified as repellents in certain vertebrates). Several behavioral parameters were measured and analyzed as the number and duration of contact with the odor, mouth openings, vocalizations (air only) and air bubble production (water only). Our results, although limited by the low number of animals monitored $(n=5)$, suggest that California sea lions are able to discriminate between different odors both in the air and under water. In the aquatic environment, the process allowing the perception of odors remains to be characterized. Applications to this work could be considered in captive conditions as well as in the wild.

Keywords (6): California sea lion, Zalophus californianus, olfaction, discrimination, aquatic environment, social/food odors 


\section{Introduction}

In pinnipeds, the auditory system is considered as highly developed. Indeed, the majority of pinnipeds vocalize in a variety of social contexts, and the importance of acoustic signals in social recognition has been demonstrated $[1,2]$. For instance, vocalizations are involved in mother-pup recognition, territorial defense and rival assessment [1,2]. Regarding olfaction, pinnipeds, like all marine mammals, have long been considered 'microsmatic', i.e., having a poorly developed sense of smell [3]. However, some studies conducted in captivity and in the wild have pinpointed that olfaction plays a significant role in regulating the behavior of certain pinniped species, and that some of these species (at least) may perceive and discriminate olfactory stimuli at very low concentrations [4,5]. Regarding mother-young recognition, the mother accepts or rejects the young after intense sniffing in various pinnipeds species $[1,6]$. It has been demonstrated in Australian sea lion (Neophoca cinerea) that mothers are able to discriminate the odor of their pup from that of another one [7].

Olfactory signals are also important in social interactions occurring during the reproductive period. Male New Zealand fur seals (Arctocephalus forsteri), for example, sniff the facial and perineal regions of females, suggesting an olfactory examination of the female's reproductive status [8]. Chemical analyses have shown that the odors of young Australian sea lions vary with age but not depending on the sex or body region. Body odors therefore appear to carry intraspecific information [9]. In addition, there is no similarity in chemical profiles of mothers and juveniles, thus ruling out the assumption of 'phenotype matching' in the process of olfactory recognition of pups by their mothers in this species [10]. However, a link between chemical profiles and genetic proximity has been found in Antarctic fur seal (Arctocephalus gazella) mother-pup pairs [11]. Moreover, it has been shown in Australian sea lions that the chemical profiles of individuals from two distinct colonies were different [10] and under the influence of the environment and/or genetic variability (even close colonies show high genetic 
variability [12]). In addition to its involvement in social behavior, olfaction seems to be used by some pinnipeds in their search for food. Thus, harbor seals (Phoca vitulina) find areas of high forage value by using odor cues for spatial orientation and food detection $[4,13]$.

Cape fur seals (Arctocephalus pusillus pusillus) discriminate between different monomolecular odors [14] and are able to memorize an odor stimulus that became significant by learning, even a few weeks after learning exposure. Another study highlighted that the sense of smell plays an underestimated role in regulating the behavior of these animals since it has been shown that these fur seals are able to discriminate between aliphatic odor [5]. A recent study also indicates that adults and sub-adults can discriminate between the odors of an adult male and a non-specific control odor, i.e., the odor of an alga present in the environment (Charrier et al., unpublished data).

While on land some pinnipeds appear to use olfaction to display a diversity of behaviors, the use of this sensory modality has never been demonstrated in the aquatic environment.

In this context of major gaps that still exist in the literature concerning olfactory capabilities of pinnipeds and their role both in the context of foraging and social interactions, the aim of our study was to test and compare, in terrestrial as in aquatic environments, the ability of captive California sea lions (1) to discriminate between food, social and repellent odors, and (2) to initiate the assessment of the use of olfaction under water. We carried out the experiments on captive animals, knowing the limits and advantages related to this context, in order to obtain results which could allow us in the future to continue this study in a natural environment and thus to have a comparative approach.

\section{Materials and methods}

\subsection{Animals and housing conditions}


Five captive-born California sea lion (Zalophus californianus) males (one intact and four castrated) living at La Flèche Zoo (La Flèche, France) were involved in our experiments. The sea lions had two separate enclosures at the zoo, one for the day and one for the night. They were locked into their indoor enclosure before the closure of the park (5:30 p.m. during the period of our study, conducted in February-March 2020). This building consists of a medical room where training and treatments were carried out as well as six individual pens. Animals went to their outdoor enclosure in the morning if the weather allowed it. Their outdoor facility comprised a concrete beach and a chlorinated pool.

In addition, the animals returned to their indoor enclosure daily in the afternoon to be taken out individually, or in pairs, to participate in training. During these daily trainings, which lasted about 20 minutes per sea lion, animals were rewarded with fish and received petting and praising from their zookeepers. During the day, animals were fed individually (in the indoor enclosure or during training) and collectively (several times a day). The food varied according to the season: fatty fish in winter (Clupea harengus) and lean fish in summer (Merlangius merlangus), but animals could also get sprat or roach in their rations. The amount of fish distributed per day varied from one individual to another (depending on their weight weekly checked).

This present study complies with the European Union Directive on the Protection of Animals Used for Scientific Purposes (EU Directive 2010/63/EU) and also with current French laws. Animal husbandry and care were under the management of the animal zookeepers of La Flèche Zoo, France.

\subsection{Odor stimuli}


The tested odors were of animal, human or vegetal origin. All were collected and presented on sterile compresses (Mercurochrome, 20x20 cm, 100\% cotton) by the experimenter or one zookeeper wearing nitrile gloves. The three types of odor used were:

- Animal odors collected a) from the California sea lions at La Flèche Zoo and from other California sea lions, castrated and intact males and females, at Beauval Zoo (France): the zookeepers rubbed the animal's head, neck and body with a sterile compress during $45 \sin$ the morning in the outdoor facility; b) from the male tiger (Panthera tigris) at La Flèche Zoo: the zookeepers rubbed during 30swith one hand the tiger's body through the enclosure fence as it approached; c) from herring (oily fish much appreciated by sea lions) and whiting (lean fish less appreciated): the experimenter rubbed the compress during 45sdirectly on thawed fish defrosted for 10 min intended for feeding the animals. - Human odors collected from two of the four zookeepers dedicated to the sea lions at La Flèche Zoo. In our first experimental session, the zookeepers rubbed a sterile compress on their own face, neck and underarms during 30s. In our second session, two of the zookeepers wore a $100 \%$ cotton white t-shirt the night before the experiment. The t-shirts were collected in the morning and kept in an individual plastic bag until the experiment (34h later). - Vegetal odors from organic essential oils of camphor (Herbes et Traditions, France) and black pepper (Pranarôm, France) known to trigger repulsion in terrestrial mammals [common vole (Microtus arvalis) and snowshoe hare (Lepus americanus)] [15,16] but also in a pinniped species, the harbor seal (Campagna S., personal observations): the experimenter poured 5 drops of essential oil on a sterile compress. 
The collected odors were either used directly in the experiments or stored in the freezer in Ziploc bags for further presentations. Freezing was considered to allow a long-term odor retention with minimal degradation $[17,18]$.

All these odors were separated into two categories: social odors (sea lion, tiger and zookeeper cues) and non-social odors (food/attractive and vegetal/repellent cues). Within the social odors, some were considered as familiar (California sea lion from La Flèche Zoo and their zookeepers), others as unfamiliar (California sea lion from Beauval Zoo, Saint Aignan, France, and the tiger from La Flèche Zoo). Among the familiar odors, those of the two zookeepers were sub-divided into "zookeeper 1", who was the first to be recruited in the aquatic team at La Flèche zoo (12 years of seniority) and "zookeeper 2", who was the last to be recruited in the team ( 3 years of seniority). Finally, the odors of the five sea lions were also divided into two categories as follows: "affinity -" vs. "affinity +" when the odor was from a group member with which the target animal displayed the least vs. the most affiliative interactions according to the zookeepers, respectively.

\subsection{Experimental set-up}

Animals were tested in two types of environment, terrestrial and aquatic. Two sessions (session 1 and session 2) were carried out, 18 days apart, in order to limit the animals' habituation to the experimental devices and testing procedure.

In the aerial environment, 14 conditions were tested during session 1: 10 conditions with one target odor vs. water (herring, whiting, zookeeper 1, zookeeper 2, familiar conspecific, unfamiliar conspecific, pepper, camphor, affinity -, tiger vs. water) and 4 conditions with two target odors (herring vs. whiting, zookeeper 1 vs. zookeeper 2, familiar vs. unfamiliar, affinity 
- vs. affinity +). The "familiar vs. water" condition was identical to the "affinity + vs. water" condition.

During session 2, 6 conditions were tested: 1 condition with a single target odor (camphor vs. water) and 5 with two target odors (herring vs. whiting, zookeeper 1 vs. zookeeper 2, familiar vs. unfamiliar, affinity - vs. affinity + , herring vs. herring + camphor). All experiments took place in the indoor enclosure, in each sea lion individual pens.

During session 1, the experimenter placed the compress impregnated with the target odor or control odor (pool water) at the end of a small metal rod $(35 \mathrm{~cm} * 5 \mathrm{~cm})$ previously cleaned with alcohol. The compress was attached to the metal rod with a clamp, which was also cleaned with alcohol. The rod was then presented to the animal through the bars of its lodge for 30 s after the animal's first contact with the compress. The animals had the opportunity of direct physical contact with the compress but they could not remove it from the rod. Each animal was tested with the two stimuli of a same condition sequentially. The order of odor presentation was random for each condition but remained the same for all animals in the session. The time interval between the two stimuli presentations was $30 \mathrm{~s}$. In session 2 , the target and control odors were presented simultaneously to the animal at the end of two identical metal rods, each odor being separated by $40 \mathrm{~cm}$. The position of the target and control odor, right or left, was randomly chosen but remained the same for all animals in the session.

In the aquatic environment, 2 conditions were tested during session 1 only, with one odor (water vs. herring, water vs. familiar). Conditions with food odors were carried out during session 2 , but unfortunately without exploitable results. The experiments took place in the outdoor pool. The protocol was the same as in the terrestrial environment, except that the animal was tested in the presence of a zookeeper for safety reasons. The experimenter stood at the edge of the pool with the metal rod just below the water surface and the zookeeper joined 
him with the animal; the zookeeper remained close to the experimenter without giving any order to the animal. We expected that sea lions would approach the device by curiosity. Their behavior was then observed for 30s after the first contact with the compress. Within a test, the time interval between the two stimuli presentations was 30s. Each condition involved the five sea lions. Regardless of the environment, individuals were tested separately one after the other in a random order, and they were tested twice a day with at least a 4-hour interval (morning and afternoon) for each session in a random environment (either in the air in the morning and in the water in the afternoon or conversely). The compresses were changed between each animal. The experimental device was cleaned with water between each animal and cleaned with alcohol between each testing condition. Animals were not rewarded following the experiment, but at the end of the session after all animals have been tested.

\subsection{Behavioral responses}

The olfactory discrimination abilities of the sea lions in both terrestrial and aquatic environments were studied by quantifying several behavioral parameters recorded during the tests: the number and total duration a) of snout contacts with the compress, b) of vocalizations (terrestrial environment only), c) of bubble production (aquatic environment only), and d) of mouth openings. These variables were quantified a posteriori, during the analysis of video sequences recorded (GoPro Hero 3 camera) throughout the duration of the tests. Each 30-sec video was analyzed frame by frame using Avidemux software.

During session 1, the camera was attached to one end of the metal rod, with the compress attached at the other end, and thus the head of the animal was clearly visible on the video. During session 2, the camera was attached to the experimenter's chest so that the two compresses were in the camera field. 


\subsection{Statistics}

The statistical analyses were performed using RStudio (R version 3.5.0). A PCA analysis was performed on our 6 behavioral parameters [number and duration of contacts, vocalizations (air only), bubble production (aquatic only), mouth openings]. This allowed us to obtain a composite score of the behavioral response for each test performed on each individual. These composite scores were then used in mixed linear models as a "response" variable, allowing us to compare the responses obtained with the control odors to those obtained with the target odors, but also to compare the responses between two target odors. The odors, the odor categories (human, animal, vegetal) and categories of odor tests (social, non-social) were coded as "fixed factors". The order of presentation and individuals were coded as "random factors" to take into account repeated measures. Interactions between these different factors were implemented in the model or removed from the model if not significant (the model was then recalculated). When a significant effect was obtained, post-hoc tests were carried out by running the same models with data subsets allowing paired comparisons and sequential Bonferroni corrections were applied to account for multiple comparisons (Holm's procedure).

\section{Results}

All the behavioural responses obtained during our different tests can be found in Supplementary Table 1.

\subsection{Tests in the terrestrial environment}

In the aerial environment, the PCA indicated that PC1 (first principal component) explained $66.3 \%$ of the variability and had an eigenvalue $>1$. The PC1 scores obtained for each test 
were therefore used for further analysis. Moreover, all behavioral variables were positively correlated to PC1 with the same magnitude (0.37 to 0.44$)$. Strong reactions were therefore represented by positive and high PC1 scores.

Analysis of the composite scores showed that the factor interaction "odor $\mathrm{x}$ test type" was non-significant $\left(\chi^{2}=2.75, \mathrm{p}=0.6\right)$, but odor $\left(\chi^{2}=114.22, \mathrm{p}<0.001\right)$ and test type $\left(\chi^{2}=90.58\right.$, $\mathrm{p}<0.001)$ showed a significant effect on the responses. Since the test type had a significant effect, the data were analyzed separately for each of them.

\subsubsection{Session 1}

Post-hoc analyses between the two odors of the same category revealed significant differences, even after Bonferroni - Holm corrections (Figure 1A, B, C).

The responses displayed by sea lions to food odors (fish) were significantly stronger than those displayed to our control (water; $\chi^{2}>35.38, \mathrm{p}<0.001$ ) (Figure 1A). Responses to human odors were also significantly stronger compared to water $\left(\chi^{2}>9.24, \mathrm{p}<0.01\right)$. Concerning responses to other animal odors, those displayed towards tiger odor did not differ significantly compared to water $\left(\chi^{2}=0.01, \mathrm{p}=0.91\right)$. For the tests involving odors from conspecifics, behavioral responses to "affinity - "were weak and not significantly different from water $\left(\chi^{2}=\right.$ 1. $72, \mathrm{p}=0.57)$ but responses to familiar and unfamiliar odors were significantly higher or tended to be higher than those to the control (familiar vs. water: $\chi^{2}=6.26, p=0.06$; unfamiliar vs. water: $\chi^{2}=15.90, p<0.001$ ) (Figure $1 B, C$ ). For plant odors, the results did not reveal any significant differences in responsiveness with the control (camphor or pepper vs. water: $\chi^{2}<3.26$, p values $\left.>0.28\right)($ Figure $1 D)$. 
251 Post-hoc analyses on PC1 scores for the two-odor tests did not reveal any significant 252 differences (affinity - vs. affinity $+: \chi^{2}=2.21, \mathrm{p}=0.28$; familiar vs. unfamiliar: $\chi^{2}=0.49, \mathrm{p}=$ 2530.49 ; zookeeper 1 vs. zookeeper $2: \chi^{2}=3.00, \mathrm{p}=0.27$; herring vs. whiting: $\chi^{2}=3.35, \mathrm{p}=$ 254 0.27) (Figure 2). However, before applying the Bonferroni-Holm correction, PC1 scores

Please, insert Figure 2 here

\subsubsection{Session 2}

Post-hoc analyses on PC1 scores (Figure 3A, B) showed that only the behavioral responses obtained with herring were significantly stronger than those obtained with herring + camphor $\left(\chi^{2}=0.02, p=0.02\right)($ Figure $3 \mathrm{~A})$.

The responses obtained for all other tests (Figure 3A, B) were not found significantly different (herring vs. whiting: $\chi^{2}=1.21, \mathrm{p}=1.00$; water vs. camphor: $\chi^{2}=0.02, \mathrm{p}=1.00$; affinity - vs. affinity $+: \chi^{2}=1.24, \mathrm{p}=1.00$; familiar vs. unfamiliar: $\chi^{2}=0.18, \mathrm{p}=1.00$; zookeeper $1 \mathrm{vs}$. zookeeper $\left.2: \chi^{2}=0.38, \mathrm{p}=1.00\right)$.

Please, insert Figure 3 here

\subsection{Tests in the aquatic environment}


272 In the aquatic environment, only 3 out of 5 sea lions approached our experimental device 273 (metal rod) and were thus included in our analysis. Two conditions were tested: water vs. 274 familiar and water vs. herring. During the odor investigations, the number and duration of

275 contacts did not vary between the target odors (familiar or herring) and the water (Figure 4A). However, while the number of contacts did not vary significantly between the herring odor and water, the duration of contacts for herring was nearly three times longer than for water (Figure 4B).

During the tests, we also observed some animals' production of bubbles and openings of the mouth. The mouth opening occurred twice during the presentation of herring odor for one individual, while the bubble production was observed for two sea lions with a total of 6 occurrences ( 3 in response to water, 2 to herring and 1 to the familiar odor).

Due to the small number of animals approaching our device $(n=3)$, no statistical tests could be done on these data. However, according to our observations and the PC scores, the behavioral responses to herring seemed to be stronger than to water (Figure 5A, B). In contrast, no clear difference was observed between the responses displayed to the familiar odor and to water.

$$
\text { Please, insert Figures } 4 \text { and } 5 \text { here }
$$

\section{Discussion}

Our study aimed to determine whether captive California sea lions displayed spontaneous behavioral responsiveness or preferences for certain odors depending on their nature/category and the medium in which the odor stimuli were presented (air vs. water). The results showed 
that California sea lions responded strongly to some odors, both in the air and under water, demonstrating effective olfactory abilities for social and non-social odors.

\subsection{Odor perception in the air}

In the aerial environment, when a fish odor - herring or whiting in our conditions - was presented sequentially to the individuals (session 1), one of the responses that we considered as behaviorally relevant, i.e., vocalizations, was significantly more often displayed by sea lions than during presentation of the control stimulus (water); this was not observed for any other odor stimulus compared to water (Figure 6).

Please, insert Figure 6 here

Sea lions appear therefore able, as expected, to selectively respond to fish using olfaction. Interestingly, the behavioral variable contributing the most to the fish vs. water discrimination was their vocal production and not sniffing (i.e., olfactory investigation) as we should have expected. Moreover, vocalizations were only produced by the sea lions during the presentation of fish odors, and can be interpreted as a high-motivation trait. According to the zookeepers, the sea lions have a preference for herring, an oily fish, over whiting, a lean fish. Although this preference did not emerge significantly in our experiments, one may note that when the two with odors were presented sequentially, vocalizations were only observed for herring. Further trials including additional individuals would thus be necessary to corroborate this preference. Such food preference illustrated by stronger behavioral responsiveness to the preferred fish smell suggests that pinnipeds could use olfactory cues during foraging activities. Laska and colleagues (2008) demonstrated that captive Cape fur seals were able to discriminate the odors of two different fish mixtures, whatever these mixtures were highly different (i.e., from different species) or weakly different (i.e., one with, one without fish oil). 
These findings clearly illustrate their sharp sense of smell. Another study, on captive harbor seals, showed a high sensitivity for atmospheric DMS, a marker of high primary productivity [4]. The perception and discrimination among fish odors in air and/or under water would thus benefit pinnipeds to orient their foraging investigations and also to identify the preferred preys during their foraging trips at sea.

Concerning the vegetal odors considered as "repellent" in other mammal species including a pinniped one, the harbor seal (Campagna S., personal observations), the group of sea lions involved in our study did not show distinct responsiveness to camphor or pepper vs. water. Camphor and pepper may not have a strong repellent value for California sea lions. Both these tested essential oils are known to have trigeminal properties in mammals. The trigeminal system is differently activated depending on the nature of the odorants: some are very active even at low concentration while others need very high concentration to activate this system [19]. When trigeminal activation occurs, odorants may generate different sensations such as " freshness" (peppermint, eucalyptus), "pain" (vinegar, acetone) or "warmth" (alcohol, cinnamon) [19,20]. These sensations are linked to specific trigeminal receptors activated by specific odorants [21]. In California sea lions, the lack of strong and clear repellent effects of camphor and pepper observed here suggests that the trigeminal system may not be activated by these odors, at least at the concentrations used in our conditions, or that the trigeminal system is not involved for olfactory assessment in this species. Further investigations using different concentrations of camphor and pepper, as additional olfactory-trigeminal odors, would be needed to conclude on the effective role of the trigeminal system on olfactory abilities in pinnipeds.

Another possible explanation of this absence of repellent effect is that the two stimuli were tested at the end of our experimental sessions. Therefore, the animals were putatively 
habituated to the procedure and this habituation effect may have impacted their responsiveness.

However, and interestingly, the results from the "fish vs. fish + camphor" condition used in session 2 highlighted that camphor may actually have a negative hedonic value in California sea lions since animals displayed a reduced response to the herring with camphor odor. Without having a powerful repellent value by itself in California sea lions, camphor essential oil could therefore have a negative effect when it is paired with an attractive food odor. This effect could be the result of interactions between molecules from the repellent and the food. Again, a more in-depth study on that question would be useful to determine the putative repellent effect of camphor in California sea lions and if this effect is effective over prolonged periods. Indeed, a recent study has shown that the use of a chemical repellent, HaTe2, spread on plants is not effective over time against a terrestrial mammal, the fallow deer (Dama dama) [22].

The use of odors as pinniped repellents could have applications in the field, particularly to prevent depredation caused by pinnipeds in aquaculture farms or in fishing traps [23], and thus to limit their impact on fisheries - an impact experienced as very negative by fishermen.

The presentation of the odor of another carnivore, here a tiger (Panthera tigris tigris), did not trigger particular responsiveness in sea lions. This clearly indicated that, in our conditions, sea lions did not react to any odors presented in contrast to water. Here, this unfamiliar animal odor did not appear to have any relevance to them. In the future, it will be interesting to test pinnipeds for their responsiveness to odors from biologically relevant species such as competitor or terrestrial predator. For instance, Cape fur seals could be tested with the odors of jackals or hyenas, known to constitute some of their terrestrial predators [24].

Within the group of sea lions monitored at La Flèche Zoo, affinities between the five animals were established and mostly based on their seniority at the zoo. While some individuals 
displayed agonistic interactions between each other, in particular the animals called Tanguy and Wally, this kind of interactions remained rare. This may thus explain the absence of significance in their behavioral responses when they were tested with both odors "affinity +" vs "affinity-". More data would be useful to further investigate the link that may exist between odor-guided behavior and degree of affinity/dominance among conspecifics in these pinnipeds.

Similarly, when tested with familiar and unfamiliar conspecific odors, the sea lions reacted significantly more often to both odors compared to water, but no significant difference was observed when these odors were presented in the same session (in sequential or simultaneous testing). This result was quite surprising as we expected that sea lions from La Flèche Zoo would have shown a stronger reaction to the odor of unfamiliar individuals for instance, but this was not the case. Social olfactory communication is used in pinnipeds in several interactions, and it has been experimentally shown that Australian sea lion mothers discriminate between the odor of their filial pup and those of non-filial pups [7]. Another study on Australian sea lions had shown that animals from two different breeding colonies presented different chemical profiles, which are related to both genetic and environmental differences [9]. Therefore, the absence of such discrimination observed in our conditions could be explained by the fact that the odors of familiar and unfamiliar individuals were considered as equally attractive to the tested animals. It is also possible that our low sample size, a loss or change in odor quality related to the freezing/thawing of our samples can have led to the lack of significant behavioral responses. However, the freezing/thawing effect is unlikely since our samples triggered animal responses when tested vs. water. Moreover, testing samples were frozen for 3-4 weeks only while other studies conducted in Australian sea lions $[9,10]$ and polar bears [18] showed a very good conservation of odors during freezing and after thawing, even after several weeks or years. 
Finally, considering the responsiveness of sea lions to human odors, our results pinpoint that animals were able to discriminate between zookeepers' odors and water, regardless of how long the zookeeper have been working at the zoo, with higher responsiveness to them. Since no significant differences were displayed in the sequential or simultaneous presentation of the odors of the two zookeepers, sea lions might not react to one zookeeper more than the other based only on the sense of smell. Nevertheless, and interestingly, even if the result was not statistically significant, animal responsiveness to zookeeper 1 (the one who has been working at the zoo for the longest time) tended to be higher than to zookeeper 2 (recently recruited) during the sequential presentation of these odors. Such responsiveness to human odors deserves also to be further assessed, for instance by comparing the odors of sea lions' zookeepers vs. zookeepers of other animals from the same zoo and/or vs. unfamiliar people (i.e., not working at the zoo). If sea lions display a positive and preferred responsiveness to their own zookeepers' odors, these stimuli could be used as positive cues to reduce the stress of the animals in certain situations such as veterinary procedures or transport.

\subsection{Odor perception under water}

In pinnipeds, the use of olfaction in the aquatic environment can not follow the same perceptual processes as in the air since the animals cannot "sniff" under water, at risk of drowning. In some other species such as the star-nosed mole (Condylura cristata) or the American water-shrew (Sorex palustris), the use of olfaction under water is based on the production of air bubbles, which are exhaled on objects of interest or on chemical tracks, and then inhaled by the animals through the nose [25]. During our underwater experiment, we observed the production of air bubbles via the nostrils during the test with herring, and also during the presentation of the familiar odor and the water control. One may therefore assume that sea lions could detect and perceive odor stimuli under water by producing air bubbles 
towards an odor stimulus, which would then be recaptured by the nostrils or the mouth.

However, during our experiment, we did not observe any case of re-inhalation of air bubbles via the mouth or the nostrils. Pinnipeds do not breathe under water, but they might exhale or inhale the bubbles produced when in contact with olfactory stimuli. Another possibility is that they might perceive the odor when the emitted bubbles pierce the surface of the water. Anyway, the interest displayed by sea lions for the herring odor in our condition pinpoints their ability to perceive certain odor cues in the aquatic environment. However, this interest might have been triggered by taste perception rather than by odor. Further investigations are required to clearly determine whether bubble sniffing could be used by some semi-aquatic mammals, such as pinnipeds.

\subsection{Issues with the method and animal welfare}

In the wild, pinnipeds show high level of curiosity for novelty, approaching and sniffing any new elements contained in the environment. This may have dramatic consequences when, for instance, it led animals to become entangled in plastic debris (fishing lines and nets, plastics...) [23]. When we started this study, we assumed that this natural curiosity of sea lions would guarantee the interest in, and approach to, our experimental devices. Surprisingly, the animals showed actually only a weak attention for our experimental devices, even though they were introduced in their daily environment. This lack of interest in exploration of new elements added in the environment may be a consequence of captivity or a consequence of a lack of interest in enrichments. To stimulate their sea lions, zookeepers from La Flèche Zoo regularly enrich the environment with ice cubes, balls, floating platform, food, but they have noticed that the animals can get bored very quickly depending on the enrichment. In this context, the use of odor enrichment may be relevant to improve attention of sea lions for novel objects, at least during the periods of the day when they are not in contact with their 
zookeepers. To this end, offering new, multi-sensory objects like floating platforms or

443 submerged structures, on which familiar sea lion sounds are broadcast and odors from 444 familiar food or social sources, or from the sea lions' natural environment are diffused could 445 be developed. Moreover, it would be interesting to test the response to novelty of captive sea 446 lions from other zoos, which have less training and/or contact with zookeepers than those 447 from La Flèche Zoo, in order to determine if the daily presence of the zookeepers reduces the attention of the animals to other stimuli than those used in the training. Furthermore, familiar or unfamiliar odors could be directly used to enrich the environment. Indeed, it was showed in California sea lions that the introduction of different odors (i.e., familiar odors: soil, sand, kelp and sardine oil; unfamiliar odors: orange, banana, vanilla extract and cinnamon) caused an increase in habitat utilization with less time spent in the water [26] and, consequently, a reduction in the majority of stereotypical behaviors [26]. A solution to enrich the environment and improve the attention of sea lions for novel objects could then be to introduce odorized objects during training periods, and to encourage zookeepers to promote interactions of sea lions with novel objects as targets to play with, in both the indoor and the outdoor environments.

Finally, the lack of differential behavioral responses towards the majority of stimuli combinations tested in this study might be the results of the experimental approach, based on spontaneous preferences and aversions. Therefore, these results do not allow us to draw firm conclusions as to the perceptual or discriminatory limits of their sensory system, but at least 462 they gave us some ideas for future directions. To deeply examine the olfactory abilities of 463 pinnipeds in-air and under water, we have for instance planned to run operant conditioning procedures that have been successfully used in pinnipeds $[4,5,14]$ and that could help us to validate our hypotheses. 


\section{Acknowledgments}

468

469

470

471

472

473

474

475

476

477

478

479

480

481

482

483

484

485

486

487

488

489

All animal zookeepers at La Flèche zoo involved in this study are gratefully acknowledged for their help and support. We would also like to thank the animal zookeepers and Baptiste Mulot at Beauval zoo for their contribution. This study was funded by CNRS (IC, GC, JB) and the CNRS-IRP program SoCNetMM "Social Communication Network in Marine Mammals".

\section{References}

[1] S.J. Insley, A. V. Phillips, I. Charrier, A review of social recognition in pinnipeds, Aquat. Mamm. 29 (2003) 181-201. https://doi.org/10.1578/016754203101024149.

[2] I. Charrier, Mother--Offspring Vocal Recognition and Social System in Pinnipeds, in: T. Aubin, N. Mathevon (Eds.), Coding Strateg. Vertebr. Acoust. Commun., Springer International Publishing, Cham, 2020: pp. 231-246. https://doi.org/10.1007/978-3-03039200-0_9.

[3] R.E. Brown, The marine mammals: orders Cetacea, Pinnipedia, and Sirenia., in: R. Brown, D. Macdonald (Eds.), Soc. Odours Mamm., Oxford: Cl, 1985: pp. 723-731.

[4] S. Kowalewsky, M. Dambach, B. Mauck, G. Dehnhardt, High olfactory sensitivity for dimethyl sulphide in harbour seals, Biol. Lett. 2 (2006) 106-109. https://doi.org/10.1098/rsbl.2005.0380.

[5] M. Laska, E. Lord, S. Selin, M. Amundin, Olfactory Discrimination of Aliphatic Odorants in South African Fur Seals (Arctocephalus pusillus), J. Comp. Psychol. 124 (2010) 187-193. https://doi.org/10.1037/a0018189.

[6] W.R. LOWELL, W.F. FLANIGAN Jr, Marine mammal chemoreception, Mamm. Rev. 
10 (1980) 53-59. https://doi.org/10.1111/j.1365-2907.1980.tb00233.x.

491

492

493

494

495

496

497

498

499

500

501

502

503

504

505

506

507

508

509

510

511

512

[7]

B.J. Pitcher, R.G. Harcourt, B. Schaal, I. Charrier, Social olfaction in marine mammals: Wild female Australian sea lions can identify their pup's scent, Biol. Lett. 7 (2010) 6062. https://doi.org/10.1098/rsbl.2010.0569.

[8] E.H. Miller, Social behaviuor between adult male and female New Zealand fur seals, Arctocephalus forsteri (Lesson) during the breeding season, Aust. J. Zool. 22 (1974) 155-173. https://doi.org/10.1071/ZO9740155.

[9] K. Wierucka, N. Barthes, B.J. Pitcher, B. Schaal, I. Charrier, R.G. Harcourt, Chemical Profiles of Integumentary and Glandular Substrates in Australian Sea Lion Pups (Neophoca cinerea), Chem. Senses. 44 (2019) 205-214. https://doi.org/10.1093/chemse/bjz008.

[10] K. Wierucka, N. Barthes, R. Harcourt, B. Schaal, I. Charrier, B.J. Pitcher, Chemical fingerprints suggest direct familiarisation rather than phenotype matching during olfactory recognition in Australian sea lions (Neophoca cinerea), J. Exp. Mar. Bio. Ecol. 517 (2019) 49-53. https://doi.org/10.1016/j.jembe.2019.06.001.

[11] M.A. Stoffel, B.A. Caspers, J. Forcada, A. Giannakara, M. Baier, L. Eberhart-Phillips, C. Müller, J.I. Hoffman, Chemical fingerprints encode mother-offspring similarity, colony membership, relatedness, and genetic quality in fur seals, Proc. Natl. Acad. Sci. 112 (2015) E5005 LP-E5012. https://doi.org/10.1073/pnas.1506076112.

[12] H. Ahonen, A.D. Lowther, R.G. Harcourt, S.D. Goldsworthy, I. Charrier, A.J. Stow, The limits of dispersal: Fine scale spatial genetic structure in Australian sea lions, Front. Mar. Sci. 3 (2016) 1-12. https://doi.org/10.3389/fmars.2016.00065.

[13] P.M. Thompson, D. Miller, Summer Foraging Activity and Movements of Radio- 
Tagged Common Seals (Phoca vitulina. L.) in the Moray Firth, Scotland, J. Appl. Ecol. 27 (1990) 492-501. https://doi.org/10.2307/2404296.

[14] M. Laska, M. Svelander, M. Amundin, Successful acquisition of an olfactory discrimination paradigm by South African fur seals, Arctocephalus pusillus, Physiol. Behav. 93 (2008) 1033-1038. https://doi.org/10.1016/j.physbeh.2008.01.019.

[15] A.R.E. Sinclair, M.K. Jogia, R.J. Andersen, Camphor from juvenile white spruce as an antifeedant for snowshoe hares, J. Chem. Ecol. 14 (1988) 1505-1514. https://doi.org/10.1007/BF01012422.

[16] A. Schlötelburg, S. Bellingrath-Kimura, J. Jacob, Development of an odorous repellent against common voles (Microtus arvalis) in laboratory screening and subsequent enclosure trials, J. Pest Sci. (2004). 92 (2019) 677-689. https://doi.org/10.1007/s10340-018-1028-3.

[17] S. Nilsson, S. Johanna, M. Amundin, C. Hartmann, A. Buettner, M. Laska, Behavioral responses to mammalian blood odor and a blood odor component in four species of large carnivores, PLoS One. 9 (2014). https://doi.org/10.1371/journal.pone.0112694.

[18] M.A. Owen, R.R. Swaisgood, C. Slocomb, S.C. Amstrup, G.M. Durner, K. Simac, A.P. Pessier, An experimental investigation of chemical communication in the polar bear, J. Zool. 295 (2015) 36-43. https://doi.org/10.1111/jzo.12181.

[19] J. Frasnelli, T. Hummel, J. Berg, G. Huang, R.L. Doty, Intranasal localizability of odorants: Influence of stimulus volume, Chem. Senses. 36 (2011) 405-410. https://doi.org/10.1093/chemse/bjr001.

[20] M. Laska, H. Distel, R. Hudson, Trigeminal perception of odorant quality in congenitally anosmic subjects, Chem. Senses. 22 (1997) 447-456. 
https://doi.org/10.1093/chemse/22.4.447.

537

538

539

540

541

542

543

544

545

546

547

548

549

550

551

552

553

554

[21] R.P. Filiou, F. Lepore, B. Bryant, J.N. Lundström, J. Frasnelli, Perception of trigeminal mixtures, Chem. Senses. 40 (2015) 61-69. https://doi.org/10.1093/chemse/bju064.

[22] R.S. Stutz, L. Verschuur, O. Leimar, U.A. Bergvall, A mechanistic understanding of repellent function against mammalian herbivores, Ecol. Process. 8 (2019) 25. https://doi.org/10.1186/s13717-019-0179-3.

[23] E.M. Jepsen, P.J.N. de Bruyn, Pinniped entanglement in oceanic plastic pollution: A global review, Mar. Pollut. Bull. 145 (2019) 295-305. https://doi.org/10.1016/j.marpolbul.2019.05.042.

[24] I. Wiesel, Killing of Cape fur seal (Arctocephalus pusillus pusillus) pups by brown hyenas (Parahyaena brunnea) at mainland breeding colonies along the coastal Namib Desert, Acta Ethol. 13 (2010) 93-100. https://doi.org/10.1007/s10211-010-0078-1.

[25] K.C. Catania, Underwater "sniffing" by semi-aquatic mammals, Nature. 444 (2006) 1024-1025. https://doi.org/10.1038/4441024a.

[26] M.M. Samuelson, L.K. Lauderdale, K. Pulis, M. Solangi, T. Hoffland, H. Lyn, Olfactory Enrichment in California Sea Lions (Zalophus californianus): An Effective Tool for Captive Welfare?, J. Appl. Anim. Welf. Sci. 20 (2017) 75-85. https://doi.org/10.1080/10888705.2016.1246362. 


\section{Figures legends}

556

557

Fig. 1: Boxplots of PC1 scores based on (A) food tests, (B) human tests, (C) animal test and (D) repellent tests with a single experimental odor during the first session in the aerial environment. The fat horizontal line indicated the median; the upper and lower limit of the box itself represented the third and the first quartiles; and whiskers indicated the maximum and the minimum value. Finally, the asterisk represented a significant difference in the behavioural responses between two tested odors (significant level at $\mathrm{p}<0.05$ ).

Fig. 2: Boxplots of PC1 scores according to the tests carried out with 2 odors during the first session in the aerial environment. The fat horizontal line indicated the median; the upper and lower limit of the box itself represented the third and the first quartiles; and whiskers indicated the maximum and the minimum value.

Fig. 3: Boxplots of PC1 scores based on (A) food and repellent tests and (B) social tests during the second session in the aerial environment. The fat horizontal line indicated the median; the upper and lower limit of the box itself represented the third and the first quartiles; and whiskers indicated the maximum and the minimum value. Finally, the asterisk represented a significant difference in the behavioural responses between two tested odors (significant level at $\mathrm{p}<0.05$ ).

Fig. 4: Barplots of (A) the numbers of contacts and (B) duration of contacts according to the tests carried out and the odors tested under water. 
578 Fig. 5: Boxplots of the (A) PC1 scores and (B) PC2 scores according to the tests carried out

579 and the odors tested under water. The fat horizontal line indicated the median; the upper and 580 lower limit of the box itself represented the third and the first quartiles; and whiskers 581 indicated the maximum and the minimum value.

583 Fig. 6: Boxplots of the numbers of vocalizations based on (A) food tests, (B) human tests, (C) 584 animal test and (D) repellent tests with a single experimental odor during the first session in 585 the aerial environment. The fat horizontal line indicated the median; the upper and lower limit 586 of the box itself represented the third and the first quartiles; and whiskers indicated the 587 maximum and the minimum value. 